Review

\title{
Phenomenology of racing and crowded thoughts in mood disorders: A theoretical reappraisal
}

\author{
Camille Piguet $^{\mathrm{a}, *}$, Alexandre Dayer ${ }^{\mathrm{b}, \mathrm{c}}$, Markus Kosel $^{\mathrm{c}}$, Martin Desseilles ${ }^{\mathrm{a}, \mathrm{c}}$, \\ Patrik Vuilleumier ${ }^{\mathrm{a}}$, Gilles Bertschy ${ }^{\mathrm{c}}$ \\ a Laboratory for Neurology and Imaging of Cognition, Department of Neurosciences and Clinic of Neurology, University Medical Center, Geneva, Switzerland \\ b Department of Neurosciences, Faculty of Medicine, Geneva, Switzerland \\ c Department of Psychiatry, University Hospital of Geneva, Switzerland
}

\section{A R T I C L E I N F O}

\section{Article history:}

Received 5 February 2009

Received in revised form 31 March 2009

Accepted 7 May 2009

Available online 9 June 2009

\section{Keywords:}

Racing thoughts

Crowded thoughts

Depressive mixed state

Mood disorders

\begin{abstract}
A B S T R A C T
Background: Racing thoughts is a frequent symptom in mood disorders, particularly mixed depressive states. This paper aims to summarize our current knowledge about its phenomenology and frequency in the spectrum of mood disorders, and to offer a new theoretical framework.

Methods: We made a selective review of original and review papers in Medline and PsychInfo database using the keywords "racing thoughts", "crowded thoughts" and "depressive mixed state" in conjunction with "mood disorders".

Results: In the context of a hypomanic state, "racing thoughts" may appear as a result from an excessive production of thoughts, moving quickly from one to the other, and generating a sense of fluidity and pleasantness. In the context of depression, "racing thoughts" are phenomenologically different and better described as "crowded thoughts": they are not only characterized by too many thoughts occurring at the same time in the field of consciousness, but perceived as unpleasant and induce the feeling that ideas are difficult to catch.

Discussion and clinical relevance: We suggest that crowded thoughts might result from the mixture of a hypomanic component, with an accelerated production of new thoughts (constituting the main source of this symptom in hypomania), and a depressive component, with a deficit of inhibition of previous thoughts (hence making thoughts crowded rather than truly racing). This distinction could help better identify crowded thoughts, and consequently depressive mixed states, which has important implications for therapeutic management. It might also help to further disentangle the psychobiological processes which contribute to the complexity of mood disorders. (c) 2009 Elsevier B.V. All rights reserved.
\end{abstract}

\section{Contents}

1. Introduction . . . . . . . . . . . . . . . . . . . . . . . . . . . 190

2. Historical and modern contributions to the concept of racing and crowded thoughts . . . . . . . . . . . . . . . . . . 190

3. Prevalence of racing/crowded thoughts in $\operatorname{mood}$ disorders . . . . . . . . . . . . . . . . . . . . . . . . . . . . . . . . . . . . . . . . 192

\footnotetext{
* Corresponding author. Laboratory for Neurology and Imaging of Cognition, Neurosciences Department, University Medical Center, 1 rue Micheli-du-Crest, 1211 Genève 4, Geneva, Switzerland. Tel.: +41 22379 5324; fax: +41 223795402.

E-mail address: camille.piguet@unige.ch (C. Piguet).
} 
4. Association of racing/crowded thoughts with other affective symptoms . . . . . . . . . . . . . . . . . . . . . . 192

5. Racing/crowded thoughts and others pathologies . . . . . . . . . . . . . . . . . . . . . . . . . . . . 193

6. Racing/crowded thoughts and depressive ruminations . . . . . . . . . . . . . . . . . . . . . . . . . . . . 194

7. Synthesis. . . . . . . . . . . . . . . . . . . . . . . . . . . . . . . . 194

8. Perspectives for future research . . . . . . . . . . . . . . . . . . . . . . . . . . 195

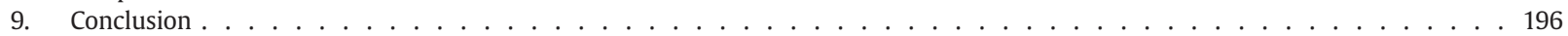

Role of funding source . . . . . . . . . . . . . . . . . . . . . . . . . . . . 196

Conflict of interest . . . . . . . . . . . . . . . . . . . . . . . . . 196

Acknowledgment . . . . . . . . . . . . . . . . . . . . . . . . . . . 196

References . . . . . . . . . . . . . . . . . . . . . . . . . . . . . . 196

\section{Introduction}

Current psychiatric research on mood disorders is rediscovering the importance of mixed states (depression with hypomanic/manic symptoms or mania with depressive symptoms), related to the idea of a continuous spectrum ranging from depression to mania (Benazzi, 2007a). A remarkable hypomanic or manic feature associated with mixed states is the subjective report of "racing thoughts".

Racing thoughts are characterized by an acceleration of thinking and reflect a pathologic increase in mental activity according to a classic model proposed by Kraepelin, exposed in the section below (Kraepelin, 1921). Racing thoughts in hypomania are classically opposed to thought inhibition in depression. However racing thoughts may be associated to depression (Akiskal et al., 2005) or mixed depression, a form of mixed state characterized by a full depressive syndrome and a few hypomanic symptoms (Benazzi, 2005d). In depression, patients with racing thoughts describe that their head is full of thoughts that they cannot stop. The terms "crowded thoughts" have therefore been proposed to label these racing thoughts in the context of depression or mixed depression (Koukopoulos, 1999). In contrast to depressive ruminations, where thoughts and ideas tend to be circumscribed to a few morbid items (Koukopoulos, 1999), crowded thoughts could be characterized by an elevated number of thoughts. We believe that studying racing/crowded thoughts could provide a good approach to tackle difficult questions concerning the frontier between unipolar and bipolar mood states, particularly the question of the nosographic status of mixed depression. A deeper understanding of this symptom could allow better clinical diagnosis, but also a better management of such patients. It has been showed indeed that psychomotor agitation in general and racing thoughts in particular are associated with a higher suicidal risk (Balazs et al., 2006) and are predictors of suicidal ideation (Benazzi, 2005c). Moreover, the usual treatment by antidepressants alone in this case could worsen psychomotor agitation and racing thoughts and are therefore not recommended (Akiskal and Benazzi, 2006; Akiskal et al., 2005).

In this paper, we seek to clarify the phenomenological concepts of racing and crowded thoughts in a clinical perspective. To this end we first review the past history and the current concepts of racing/crowded thoughts and their boundaries with depressive ruminations. We then examine their prevalence rate in mood disorders and their association with other affective symptoms on the basis of phenomenological studies. We will also consider whether racing/crowded thoughts could occur in nonmood disorders. Finally we propose a new theoretical model that integrates a clarified version of the concept of racing/crowded thoughts and that open the way for experimental investigations using cognitive paradigms.

\section{Historical and modern contributions to the concept of racing and crowded thoughts}

The dichotomy between melancholia (depression) and mania existed already in the antiquity and has been described with consistency since then. It is only in the 18th century that it has been recognized as a specific entity that became manicdepressive illness with Kraepelin at the turn of the 20th century (for more details Goodwin and Jamison, 2007). In the sixth edition of his textbook he suggested an integrative view about manic-depressive illness, emphasizing that both states could occur in a given individual at the same time. This perspective gave birth to the concept of mixed states and suggested the unity of the two pathologies. Kraepelin (1921) and his student Weygandt (in "Über die Mischzustände des manisch-depressiven Irreseins" (Salvatore et al., 2002) published almost at the same time in 1899 the famous definition of a manic-depressive spectrum, comprising mixed state, where they described three distinct areas of psychic functioning: "affect, psychomotor activity, associative thinking". Each of these functions could be excited or depressed, independently from the others. This allowed eight different combinations $(2 \times 2 \times 2)$. Two combinations were homogeneously either excited or depressed, and corresponded to pure states of mania and depression, respectively. The six remaining combinations were heterogeneous and corresponded to six different subtypes of mixed states: manic stupor, unproductive mania, manic arrest, furious (or depressive) mania, agitated depression, and depression with flight of ideas (Table 1).

One of the arguments to support their model of mixed states was the observation that some patients would complain of or show accelerated thinking, that is, an excitation in the domain of "associative thinking", not only during mania but also during depressive episodes. Weygandt (Salvatore et al., 2002) actually differentiated very clearly between disorders of speech and thoughts (e.g.: speech may be activated and thoughts inhibited when patients repeat the same sentence for hours) but he did not distinguish between accelerated thought and flight of ideas. He cited however Richarz who describes in 1858 the form of "Melancholia agitans" where patients "cannot focus their thoughts; as soon as they form an idea, it disappears, and a completely different one quickly takes its place". Richarz thought that this was 
Table 1

Classification of «mixed states» based on Weygandt and Kraepelin, Manic-depressive insanity, 1921.

\begin{tabular}{|c|c|c|c|}
\hline & Intellect (associative thinking) & Emotion (mood, affect) & Volition (psychomotor activity) \\
\hline Mania & $\uparrow$ & $\uparrow$ & $\uparrow$ \\
\hline Depressive or anxious mania/furious mania & $\uparrow$ & $\downarrow$ & $\uparrow$ \\
\hline Mania with poverty of thoughts/unproductive mania & $\downarrow$ & $\uparrow$ & $\uparrow$ \\
\hline Excited/agitated depression & $\downarrow$ & $\downarrow$ & $\uparrow$ \\
\hline Inhibited mania/manic arrest & $\uparrow$ & $\uparrow$ & $\downarrow$ \\
\hline Depression with flight of ideas & $\uparrow$ & $\downarrow$ & $\downarrow$ \\
\hline Manic stupor & $\downarrow$ & $\uparrow$ & $\downarrow$ \\
\hline Orthodox depression & $\downarrow$ & $\downarrow$ & $\downarrow$ \\
\hline
\end{tabular}

something different from the flight of ideas of manic patients, but it was not very relevant to Weygandt.

Kraepelin on his side described clinically different types of racing thoughts and considered those occurring during depression as part of a mixed state (depression with flight of ideas, depressive mania): "Patients complain that they "have so many thoughts in their head", that they cannot pray, cannot work, because other thoughts, "interpolations," come between, that they have "no settled thoughts," that they have to think of everything possible." (Kraepelin, 1921). Even in the description of "Melancholia simplex" he reported that the patient may sometimes complain "that fresh thoughts are always coming to him, that he has too much in his head, that he finds no rest, is confused". Here are some more examples out of Kraepelin: "I can't grasp all the thoughts which obtrude themselves"; "It is so stormy in my head"; "Everything goes pell-mell" - which all echo our own modern sampling of some depressive patients: "It is very crowded up there"; "It is like a formula 1 in my head". However, Kraepelin did not discriminated flight of ideas from racing thoughts. Later authors such as Lewis in the 30s ( cited by Goodwin and Jamison, 2007) and Ianzito in the 70s (Ianzito et al., 1974) agreed with the fact that racing thoughts could happen during depression.

In the 80s, Braden and Qualls (1979), interpreting Kraepelin, formally differentiated "speeded or racing thought" as "the subjective equivalent of flight of ideas", making one symptom the observable component of another process most typically associated with mania. This view was also endorsed by Goodwin and Jamison in the two editions, at a 20 years interval, of their authoritative textbook (Goodwin and Jamison, 1990, 2007). Braden and Qualls advocated that racing thoughts may not only be seen in mania, but also arise in bipolar depression, unipolar depression, cyclothymia, as well as in depression after a schizophrenic episode. They proposed that, as in flight of ideas, patients have troubles to describe the content of their thoughts "as if each thought was attended for only a brief time". They also reported striking descriptions in relation to circular themes. Two years later Braden and Ho (1981) assessed thought speed and ideomotor pressure with a self-made questionnaire. There were no consistent differences in the descriptions of racing thoughts given by manic as compared to depressed patients; but depressed patients were more likely to describe time as being perceived too slow and racing thoughts as being unpleasant.

During the same period, the AMDP-system was developed by the German school for a very precise and in depth evaluation of psychiatric symptoms (Arbeitsgemeinschaft für Methodik und Dokumentation in der Psychiatrie (AMDP), 1995). For the item 22 on "flight of ideas" in the English version (Association for Methodology and Documentation in Psychiatry (AMDP), 1982) it was specified that "accelerated thinking without a flight of idea should be rated under item P3 ("Accelerated Thinking")", a reserve item; whereas in the French version (Association pour la méthodologie et la documentation en psychiatrie (AMDP), 2000), the opposite was specified: flight of ideas sometimes occurs without an acceleration of thought. The fact that these two phenomena are different was thus clearly recognized. The French version also contains a note mentioning that accelerated thinking should be quoted under a specific item R4 "pensée accélérée". Therefore, both English and French versions added a reserve item related to "accelerated thinking", while the German version had no special quotation: acceleration of thoughts must be included in the items "increased drive" and "logorrhea".

The term "racing thoughts" also appeared in the DSM-III in 1980, but only in the criterion B3 of mania: "flight of ideas or subjective experience that thoughts are racing" (American Psychiatric Association, 1980). Since then the wording has stayed the same. It became the criterion B4 in the DSM-III-R, and the last version of DSM-IV-TR (American Psychiatric Association, 2000) gives a more specific description in manic and hypomanic episodes: "The individual's thoughts may race, often at a rate faster than can be articulated", "This experience resembles watching two or three television programs simultaneously". The flight of ideas is described by a logorrhea (which is also considered by itself in the criterion B3), with abrupt changing of topics, such that the speech can be disorganized and incoherent. It is uncommon and transitory in a hypomanic state. The ICD-10 (World Health Organization, 1994) considers only the flight of ideas in the manic episode with psychotic symptoms. Of course, manic/hypomanic symptoms are included in other disorders like schizoaffective disorder bipolar type, or cyclothymia and bipolar mixed state.

At the turn of the new millennium, Koukopoulos (1999) further supported the distinction between depressive patients with racing thoughts and manic patients with flight of ideas: racing thoughts in depressive patients are not expressed by a pressured speech and are considered as unpleasant. In Koukopoulos' opinion, they are also qualitatively different: whereas in mania thoughts tend to form a string of ideas, in depression "the phenomenon could be called crowded thoughts; the patient complains that his or her head is full of thoughts of all kind, ..., but prevalently trivial thoughts of little significance for the patient." He was thus the first to introduce the term of crowded thoughts to differentiate racing thoughts 
in depressive patients from racing thoughts in manic/hypomanic patients.

In recent years, Benazzi (2003, 2005d) contributed very actively to the concept of mixed depression and thus on racing thoughts in depression. He made a distinction between subjective racing thoughts and flight of ideas, following the DSM-IV (cf above). He also followed the distinction of Koukopoulos between racing and crowded thoughts, yet with some nuances: "In racing thoughts the head is full of rapid thoughts that the patient cannot stop, while in crowded thoughts the head is full of non-rapid thoughts that the patient cannot stop" (Benazzi, 2003). Two years later he again described crowded thoughts as "continuous flooding of the mind by ideas that the person could not stop" and racing thoughts as "increased speed of thinking" (Benazzi, 2005c,d). Following previous suggestions, he also introduced a notion of a grading (Benazzi, 2003) which has become more precise in his latest publications (Benazzi, 2005d, 2007a): "Disordered thinking in hypomania included creative thinking, crowded thoughts (non-stop thinking), racing thoughts (speedy thinking), and flight of ideas (quick, disconnected thinking)", with an emphasis on the grading of symptoms in this order.

In line with the original Kraepelinian model, all these processes constitute a symptom of mental overactivity, found in bipolar disorders (Benazzi, 2005d), and considered as one of the most common manic symptoms in mixed depressive state. More recently, when describing mixed states in the new version of their book, Goodwin and Jamison also introduced the term crowded thoughts in reference to Koukopoulos, Benazzi and Akiskal. But in all clinical descriptions illustrating this topic in their textbook, there was only one patient who uses this term: "all problem of the universe came crowding into my mind". Otherwise they still considered racing thoughts as the subjective counterpart of the flight of ideas (Goodwin and Jamison, 2007).

Therefore, up to now, the concept of racing thoughts has never been entirely clarified, and its nosological position still remains unsettled. At best, some authors consider it as a subjective equivalent of flight of ideas, but many clinical descriptions reviewed above strongly suggest that the phenomenon is more complex.

\section{Prevalence of racing/crowded thoughts in mood disorders}

Racing thoughts is a symptom very frequently encountered in mania, and usually not differentiated from flight of ideas. Thus, as discussed elsewhere in this paper, in mood disorders, flight of ideas is always associated with racing thoughts, but the reverse is not true: subjective racing thoughts may be present without objective flight of ideas. Rather, it is in the field of schizophrenia that the concept of flight of ideas has been developed in a way that does not involve a systematic association with racing thoughts. Hence, in the field of mood disorders, considering the prevalence rate of flight of ideas instead of racing thoughts does not inflate the prevalence rate of racing thoughts.

Goodwin and Jamison report a synthesis of 12 studies on cognitive symptoms during mania, with samples varying from 9 to 316 patients and performed between 1965 and 2005: the mean prevalence of "flight of ideas/racing thoughts" is $76 \%$ with a 41 to $100 \%$ range (Goodwin and Jamison, 2007). Other recent studies have confirmed this (Henry et al., 2003; Sato et al., 2002b). In one study (Sato et al., 2002b) the prevalence of flight of ideas/racing thoughts was higher in pure mania (76\%) than in dysphoric mania (62\%). The question of the prevalence of racing thought in hypomania is of particular interest. In a study (Benazzi and Akiskal, 2003) using the Mood Disorder Questionnaire to evaluate past hypomania, racing thoughts were the most common symptom (65.1\% rate) followed by increased energy and social activity (56\%), as well as irritability (54.6\%).

In the field of mixed episodes, Benazzi proposed the concept of dysphoric hypomania: a full DSM-IV hypomanic episode, with irritable mood, associated to a full DSM-IV major depressive episode (Benazzi, 2007b). He observed that racing/crowded thoughts were present in $97.3 \%$ of cases of dysphoric hypomania whereas it was present in $66.4 \%$ of past typical hypomanic episodes of the same bipolar II patients (typical because there were no associated major depressive episodes).

The prevalence of racing thoughts in depression remains an open question. Braden drew attention to the presence of this symptom among psychiatric inpatients, including depressed patients. A quantitative study found that more than $50 \%$ of patients experienced some form of racing thoughts, and 39\% have "persistent racing thoughts" (Braden and Ho, 1981). Concerning depressed patients specifically, on the one hand, some studies suggest very low rates of speeded thoughts, for example 5\% in one study using the Present State Examination (Ianzito et al., 1974). Interestingly, however, this study reported a very high prevalence of "thoughts confused" that could be related to crowded thoughts. A prevalence of $6 \%$ is reported in another study using the AMDP system and looking at racing thoughts in unipolar depressed patients, with this frequency increasing respectively to 12 and $14 \%$ in bipolar II and bipolar I depressed patients (Sato et al., 2003). A very low rate of "thoughts racing" was found in unipolar MDD inpatients ( 1 out of 372 ) by another study using some items of the OPCRIT (operational criteria for psychotic illness checklist: (Serretti and Olgiati, 2005)). On the other hand, an early study in 1980 showed more than $50 \%$ of moderate to severe racing thoughts in depressed patients and concluded that "in the case of primary depression, the unipolar-bipolar classification does not help identify patient groups with and without racing thoughts" (Kirstein and Smith, 1980).

Regarding the recent publications by Benazzi, a very sensitive screening of racing thoughts was applied to his secondary care out-patients. The concept of racing thoughts was extended to the concept of crowded thoughts, and the assessment included questions such as "Is your mind full of ideas you cannot stop?", "Do you think continuously?", or "Do you go to bed and still continue to think?" Thus, this assessment focused on the continuous flow of ideas, rather than the speed or the disconnection of ideas (Benazzi, personal communication). It is therefore not surprising that a high prevalence of racing/crowded thoughts was noted during Major Depressive Episode, around 55\% for unipolar patients and around 75\% for Bipolar II patients (Benazzi, 2001, 2002, 2005a,d, 2006, 2007b,c; Benazzi and Akiskal, 2001).

\section{Association of racing/crowded thoughts with other affective symptoms}

Using principal component analysis of a range of clinical features in manic patients, racing thoughts were found to be 
associated with several classical symptoms of psychomotor activation. In a first study of manic patients investigating both manic (10 items) and depressive (10 items) symptoms, a racing thought item was included in a factor "agitation", which was the second out of a five factor solution (the four remaining factors being labeled: "depression", "sleep-energy", "emotional and physical depletion", and "irritability-poor judgment"). In this "agitation" factor, the item racing thoughts was associated with accelerated speech, hyperactivity, elated mood, augmentation of activities, unusual energy, and megalomania (Hantouche et al., 2006). In a second study in manic patients, the principal component analysis resulted in a five factor solution ("dysphoric mood", "psychomotor acceleration", "psychosis", "increased hedonic function" and "irritable aggression") in which racing thoughts was included in the factor "psychomotor acceleration", together with items of pressured speech, increased motor activity, and increased contact (Cassidy et al., 1998). In a third study examining both depressive and manic symptoms during acute mania the results were somehow different: in the seven factor solution ("depressed mood", "irritable aggression", "sleep disturbance”, "psychomotor/thought inhibition", "mania", "emotional lability/agitation", and "psychosis"), racing thoughts did not appear in the "emotional lability/ agitation" factor (comprising emotional lability, distractibility, motor restlessness, and agitation) but in the mania factor together with exaggerated self esteem, euphoria, excessive social contact, flight of ideas, increased drive, and delusion of grandiosity (Sato et al., 2002a).

A slightly different aspect was found in a study on the structure of hypomanic symptoms (past hypomania in depressive outpatients): racing thoughts were not included in the first factor labeled "energized-activity" (increased activity and energy, and decreased need for sleep) but in the second and last factor labeled "irritability-thought racing", with irritable mood and distractibility (Benazzi and Akiskal, 2003). Further, another study on the structure of affective symptoms in a sample of healthy young adults found the item "my thoughts raced through my mind" to be significantly loaded on a factor “typical hypomania" (Meyer and Hautzinger, 2003), whereas the question "I was easily distracted and constantly lost my flow of thoughts", accounting for distractibility, referred to a dysphoria-depression dimension, that also comprised irritability. Taken together, these studies broadly support the notion that racing thoughts are often associated to cognitive excitation and constitute a classical symptom of mania/hypomania.

By contrast, studies using principal component analyses of manic and depressive symptoms in depressed patients are rare. In one study, a six factor solution revealed five depressionrelated factors and one general hypomanic factor which comprised racing thoughts together with flight of ideas, logorrhea, aggression, excessive social contact, increased drive, and irritability (Sato et al., 2005).

Another study, performed by our group (Bertschy et al., 2007), assessed depressive and manic symptoms in a sample of patients including cases of major depression, mixed episodes, and mania. Not surprisingly, flight of ideas was included among the typical manic items in a large first factor, with a bipolar structure opposing typical manic and depressive symptoms; whereas distractibility and concentration difficulties were part of the second factor labeled "dysphoria". The third and last factor was limited to sleep symptoms
(Bertschy et al., 2007). Benazzi also explored the contribution of racing thoughts to the delineation of mixed depression. He reported that the presence of racing thoughts has a sensitivity of $88 \%$ and a specificity of $54.6 \%$; a positive predictive value of $66.1 \%$ and a negative predictive value of $82 \%$ for the diagnostic of depressive mixed state, as defined by the association of a major depressive episode with three hypomanic symptoms (Benazzi and Akiskal, 2006). Most common manic symptoms found in unipolar depression include psychomotor agitation, irritability, and distractibility (Serretti and Olgiati, 2005), often joined by racing thoughts (Akiskal et al., 2005; Benazzi and Akiskal, 2001; Sato et al., 2003). We see that racing thoughts are not uncommon in depression, and usually associated with others symptoms of "agitation".

\section{Racing/crowded thoughts and others pathologies}

In both major classifications, DSM-IV and ICD-10, the concept of racing thoughts is not mentioned in any other categories of mental pathology, although some could arguably include related symptoms. However, the terms racing/crowded thoughts are not only used in the context of mood disorders. For instance, we have noticed that patients would use the words racing thoughts in self reported panic attacks or obsessivecompulsive disorders. We can understand this because acceleration of thoughts may be a very common human experience in the context of stress and thus considered as a subjective concomitant of arousal. But such broadening might question the specific relevance of this symptom to the field of mood disorders, perhaps like the reported emotion of anxiety or anger that are common from the perspective of subjective human experience but very unspecific in psychopathology. Although it would be beyond the scope of the present paper to thoroughly review these questions, it is necessary to consider some frontiers and possible relationships with others psychiatrics conditions.

Borderline personality disorders represent an important domain close to the field of mood disorders. We found one study that reported $92 \%$ of racing thoughts in borderline women, but the patients were all incarcerated in maximumsecurity hospitals, thus probably not very representative of all patients with borderline personality disorders (Coid, 1993). Such a result tends to support the hypothesis that the affective lability of borderline patients would resemble the mood cyclicality in bipolar patient. On the other side, some studies tend to show that, although they may share some common symptoms, both of these pathologies are separated (Benazzi, 2008; Henry et al., 2001). Unfortunately, these studies did not specifically assess racing thoughts.

Attention deficit/hyperactivity disorder (ADHD) might also be a close diagnostic neighbor, and we could intuitively consider a tight link between deficit of attention and racing thoughts. However, modern classifications (American Psychiatric Association, 2000; World Health Organization, 1994) do not mention racing/crowded thoughts as a symptom of ADHD, but emphasize excessive talkativeness instead. Nevertheless, several studies (Nierenberg et al., 2005; Wingo and Ghaemi, 2007) suggested a close relationship between both disorders, with one of them showing that flight of ideas or racing thoughts are present in about $60 \%$ of adults with ADHD and bipolar disorder, against only $20 \%$ for adults with ADHD 
only (Wilens et al., 2003). This pattern therefore indicates a predominant link of this symptom with the additional pathology, rather than ADHD itself. This may accord with another study in prepubertal and early adolescents, where the flight of ideas/racing thoughts dimension was found to represent a discriminating factor between bipolar subjects and ADHD or control (Geller et al., 2002).

Finally, another field concerned with formal thought disorder is the domain of schizophrenia. The distinction between schizophrenia and bipolar disorders has been clarified since the seminal contribution of Kraepelin. Yet, recent research, particularly in genetics, has begun to challenge the Kraepelinian dichotomy. Moreover, to some extent, some symptoms may be similar although globally manic patients present more rapid and complex speech, related to their mood tone, whereas schizophrenic patients present more disorganized and poorer speech (Goodwin and Jamison, 2007). Nevertheless, in this context, it is important to stress that some confusion may arise around the term of flight of ideas. As mentioned before, during the 80 s, it was clarified that, in the field of mood disorders, racing thought could be considered as the subjective equivalent of the flight of ideas, this latter being the visible part for the interviewer of the activated mental processes at the origin of racing thoughts. This perspective is different from the perspective of the flight of ideas in schizophrenia, for which Andreasen (1979) used the concept of "derailment". In schizophrenia, what is central is the loosening of the degree of association between thoughts, not their acceleration. Andreasen stressed that derailment in schizophrenia is a "slow, steady slippage", whereas in mania "flight of ideas is a derailment that occurs rapidly in the context of pressured speech". There is an important qualitative difference between both classes of pathology concerning the clinical presentation of their thought disorder.

\section{Racing/crowded thoughts and depressive ruminations}

Depressive ruminations are intimately associated with depressive mood (Papageorgiou, 2004), although they are not always present. Patients with depressive ruminations report repetitive thoughts about a single theme, or sometimes a few, always concerning their present distress and the circumstances surrounding their sadness (Conway et al., 2000). These repetitive thoughts are typically focused on the causes, meanings, and consequences of depressive symptoms (NolenHoeksema, 1991). Patients with ruminations seem able to report their worries, contrarily to patients with crowded thoughts who are overwhelmed by numerous ideas with many different themes and feel unable to "catch their thoughts". Therefore, racing thoughts in depressive mixed sates are clearly to be distinguished from the classic notion of ruminations.

\section{Synthesis}

In summary, there is now a large consensus to define racing thoughts as a subjective sensation of accelerated thinking. These can be distinguished from the flight of ideas which is generally viewed as an external manifestation of accelerated thinking. It is also clear that both phenomena do not systematically co-occur: some patients may complain of subjective racing thoughts without exhibiting any apparent flight of ideas. These differences can be illustrated by a schematic model depicted in Fig. 1 (see points a and b). These distinctions are also compatible with a gradation from racing thoughts to flight of ideas, related to the severity of manic symptoms: racing thoughts without flight of ideas may be more characteristic of hypomania (alone or associated to depression), whereas flight of ideas may be more commonly encountered during manic episodes. Although it is convenient as a general heuristics, we acknowledge that this view might include some shortcomings in special cases: e.g., patients may present an episode of mania with mutism, possibly related to catatonia (Fink and Taylor, 2001; Kraepelin, 1921), but subsequently recall intense racing thoughts.

Flight of ideas associated to racing thoughts may give rise to a pressure of speech, although pressure of speech itself may occur independently from flight of ideas as already advocated by Weygandt. For example, this would refer to patients who are restlessly repeating the same thing (see Fig. 1, point c). Flight of ideas without accelerated thinking is probably not encountered, unless we define flight of ideas as a loosening of associations as can be observed in schizophrenic patients (see Fig. 1, points d).

Our review of the literature reveals that racing thoughts with or without flight of ideas may occur in different contexts of affective disorders. In particular, they are found with a very high frequency in hypomanic and manic states. But in major depression, such phenomenon could also be present with a high frequency when there is an associated hypomanic syndrome (concept of mixed depression). However, very large differences in prevalence rates of racing thoughts have been found between

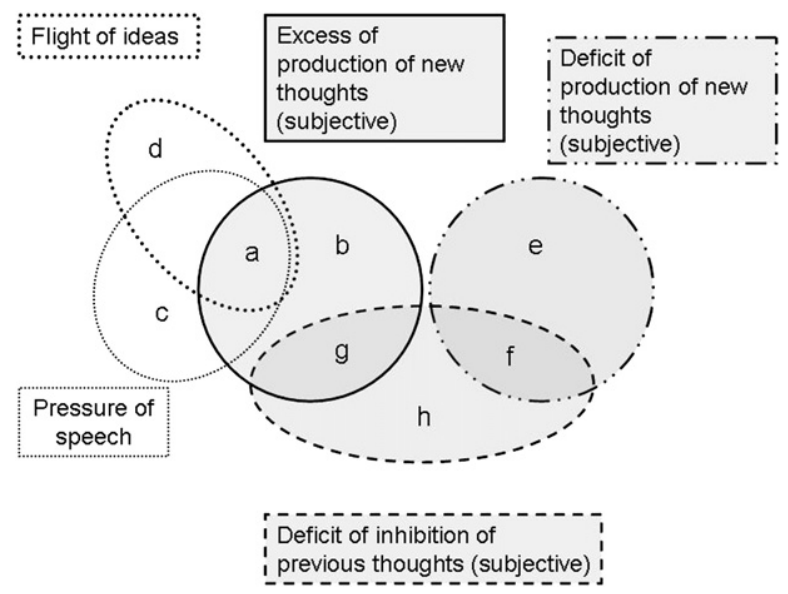

Fig. 1. A diagrammatic representation of the racing and crowded thoughts concepts and other related concepts. a: linear racing thoughts with flight of ideas (viewed as the observable component of racing thoughts) and pressure of speech (as seen in mania rather than in hypomania). b: linear racing thoughts, without flight of ideas and without pressure of speech (as seen in hypomania rather than in mania). c: pressure of speech without accelerated thinking or flight of ideas (as seen in paranoia). d: flight of ideas (ideas are disconnected) but without acceleration (subjective or objective); very close to loosening of associations (as seen in schizophrenia). e: deficit of production of new thoughts (psychic retardation) (as seen in inhibited depression without ruminations). f: deficit of production of new thoughts (psychic retardation) with deficit of inhibition of previous thoughts resulting in ruminations (as seen in inhibited depression with ruminations). g: acceleration of thinking concomitant with a deficit of inhibition of previous thoughts resulting in crowded thoughts (as seen in mixed depression). h: persisting thinking without any excess or deficit of production of new thoughts (close to the concept of fixed ideas in obsessive compulsive and related disorders). 
studies (see above). Although this is likely to result from differences in clinical assessment methods, these differences themselves also reflect the specific phenomenology of racing thoughts when they occur in the context of a depressive episode. Thus the highest rates in depression are obtained by using an assessment method that includes the concept of crowded thoughts. A more careful discrimination between racing thoughts versus crowded thoughts would therefore be of great clinical interest and should be further explored in the future.

In manic patients, racing thoughts take the form of a continuous string of ideas. Thus, one idea chasing the other represents the typical form of hypomanic racing thoughts. We propose to call this form "linear racing thoughts". In depression, in contrast, clinical descriptions indicate that many thoughts tend to be perceived as present at the same time, which is thus better described by the metaphoric term of "crowded thoughts" (Koukopoulos, 1999; Kraepelin, 1921). This high number of thoughts could provide the cornerstone for distinguishing crowded thoughts in depression from depressive ruminations. Based on our clinical experience the distinction between hypomania-related linear racing thoughts and depressionrelated crowded thoughts has undoubtedly a very high validity. Nevertheless, the phenomenology of racing versus crowded thoughts has been little studied so far. In the literature reviewed above, only definitions are offered, often supported by a few clinical illustrations, and proposed by authoritative experts of the field (Koukopoulos, Benazzi, Akiskal or Goodwin and Jamison), but specific and systematic studies are still lacking. Notably it remains to be investigated if crowded thoughts are more frequent in BP-II disorders and racing thoughts in BP-I. This differentiation might be useful in terms of diagnosis, but also for the prognosis or the choice of treatment. Indeed we know that mixed states have a more severe outcome (Keller et al., 1986) and are at higher risk of committing suicide (Balazs et al., 2006), which seem to be related to racing/crowded thoughts and psychomotor activation (Akiskal and Benazzi, 2006). It has also a potential therapeutic importance, notably concerning the adequate use of antidepressants and mood stabilizers. As suggested by our and others' clinical experience (Akiskal et al., 2005; Benazzi, 2005b,c, 2007a; Bertschy et al., 2008; Koukopoulos, 1999), a significant number of mixed depressions occur during antidepressant treatments and the therapeutic management of these mixed patients is difficult.

Consequently, several important points are waiting for clarifications. Are crowded thoughts rapid or slow or a mixture of both? Are these two subtypes of racing thoughts (linear and crowded) mutually exclusive? In our experience, some patients may pass from one subtype to the other, even from moment to moment, yet it is not clear whether both categories could actually coexist under the form of an intermediate subtype. The same questions about possible transitions and intermediate forms may also be raised for the distinction between crowded thoughts and ruminations.

More generally, the different dimensions of thoughts disorders across pathologies deserve to be elucidated in a more detailed fashion.

\section{Perspectives for future research}

In the following section we suggest a tentative theoretical framework for the differentiation of racing versus crowded thoughts. The aim of this approach is to offer a new basis for further empirical testing, through neuropsychological, genetic, or neuroimaging techniques. In this perspective, we also consider a few leads concerning the possible neuroanatomical substrates underlying these thought disturbances.

Why should racing thoughts be "crowded" rather than "linear" when they are associated to depression? Crowded thoughts may be conceptualized as a pathological thought process characterized by the occurrence of too many thoughts that co-exist almost simultaneously in consciousness, and that give to the subject a sense of constant and unpleasant agitation in his/her own thinking. In contrast, racing thoughts may be characterized by an increased speed in the succession of thoughts, and engender the subjective impression of a rapid, relatively fluid and pleasant, thinking. The common feature of racing/crowded thoughts would thus be an excess of new thoughts. However, in the context of hypomania, it might then be easy for the patient's mind to abandon a new idea so as to focus, even very transiently, on the next one (one idea chasing the other); whereas in the context of depression it might be difficult for the patient's mind to abandon a current idea to focus on the following one. The consequence of the latter is that a new idea might appear to mix with many others that remain difficult to chase or tend to recur. Put in more trivial words, it is as if crowded thoughts could be produced by the collision of two phenomena: an excess of thoughts entering into consciousness and a deficit of thoughts exiting consciousness.

In a more theoretical perspective, crowded thoughts would therefore result from the convergence between accelerated thinking at the cognitive level (generation of many new thoughts) and impaired inhibition of those newly produced thoughts, possibly implicating some mechanism that is similar to that producing ruminations (see Fig. 1, points e, f and g). Both racing and crowded thoughts may arise due to an acceleration of thoughts, but the latter would be associated with an additional deficit of inhibition of these previous new thoughts, unlike linear racing thoughts. Thus, an increased flow of new thoughts without deficit of inhibition could lead to the linear racing thoughts of hypomania; whereas an increased flow of new thoughts with deficient inhibition could lead to crowded thoughts as seen in mixed depression. In contrast, decreased new thoughts with a deficient inhibition may lead to depressive rumination; but a decreased flow of new thoughts without any

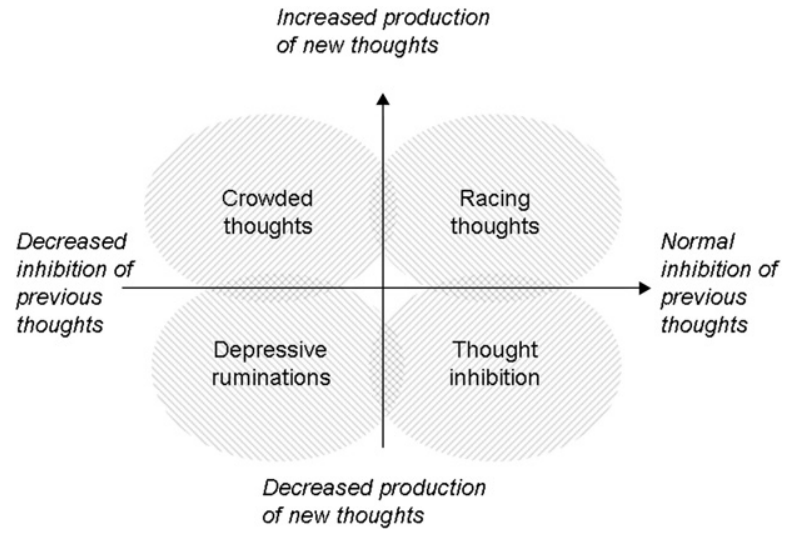

Fig. 2. A new conceptualization of racing and crowded thoughts. 
deficit of inhibition may correspond to a depression with thoughts inhibition (see Fig. 2).

The classic model proposed by Kraepelin included three distinct areas of psychic functioning ("affect, psychomotor activity, associative thinking"), each of them susceptible of being excited or depressed independently from the others. Our model differs from Kraepelin's model because we propose two (not one) cognitive processes that could be inhibited or excited, each independently from the other: associative thinking (i.e. the ability to switch to/produce new thoughts) and thinking disengagement (i.e. the ability to inhibit the processing of previously relevant information). In a more clinical perspective, we propose the existence of an interaction between a depressive element (deficit of inhibition of previous thoughts) and a manic element (excess of production of new thoughts), and that this interaction can result in a specific by-product: the phenomenon of crowded thoughts. This has some analogy with a previous construct proposed by our group: the dysphoric syndrome (irritability, inner tension, impulsivity, painful hypersensoriality), frequently associated to mixed states (Bertschy et al., 2008), which was similarly proposed to emerge through an interaction between thought excitation and depressive mood (Bertschy et al., 2007). Moreover, irritability and crowded thoughts are very frequently associated.

These more basic elements of associative thinking and thinking disengagement might then become more readily dissociable for direct empirical testing. These two components of the control of mental processes could presumably be investigated using experimental cognitive paradigms such as switching, mental flexibility, fluency, or response inhibition. Indeed a recent study examined a task switching paradigm in correlation with scores on a ruminative scale with healthy subjects, and showed that the higher people score on this scale, the more difficulties they have to inhibit a mental set to switch to another (Whitmer and Banich, 2007). Therefore we believe that these two components of thought processes should be more systematically tested in relation to thoughts disorders in patients with affective illnesses.

If crowded and racing thoughts are distinct processes, they might also have distinct neuroanatomical substrates. Based on an analogy with hypo- and hyperkinetik neurological pathologies (Aron et al., 2003; Dale et al., 2007; Hayes et al., 1998; Yehene et al., 2008), which may include thought anomalies (Leigh et al., 1987; Sachdev, 1993), and the emergent literature on neuroimaging of thoughts disorders (Gu et al., 2008; Guehl et al., 2008; Saxena and Rauch, 2000), we hypothesize that the process of inhibition of previous thoughts, which is impaired in crowded thoughts but not in racing thoughts, might be mediated by executive and limbic loops passing through the basal ganglia. In parallel, the manic component, hyperproduction of thoughts, could result from hyperactivation of the orbitofrontal cortex, leading in turn to hyperactivation of the ventral striatum (Clark and Sahakian, 2008; Lawrence et al., 2004). On the other hand, depression may lead to a deactivation of pre-frontal cortex, hence removing control over the basal ganglia. This could presumably lead to general overall inhibition (Drevets et al., 2008; Mayberg, 2003; Mayberg et al., 1999). Patients with mixed depressive states might possibly show anomalies in both of these fronto-striatal loops, whose integration in the critical "hot spots" (Haber, 2008) of the basal ganglia could thus be impossible. It is tempting to speculate that an imbalance between the two loops might conduct to difficulties in inhibiting previous thoughts, and thus lead to a clinical presentation of crowded thoughts. However, these neuroanatomical hypotheses need to be further supported by direct experimental investigations.

\section{Conclusion}

Racing thoughts and crowded thoughts are frequent symptoms in affective disorders. Their definition and classification by experts support a specific relationship between racing thoughts and hypomania/mania, on the one hand, and between crowded thoughts and mixed depression/depression, on the other hand. Yet, their phenomenology should be better documented. Here we have proposed a theoretical model pointing to a distinction between racing and crowded thoughts that could allow further clinical and experimental investigation, and thus help better understand the complex interplay between unipolar and bipolar phenomena. This is not just for the pleasant achievement of more or less perfect classifications. This is also because such a refinement in appreciating the cognitive underpinnings of mood disorders may also have an impact on the outcome and management of such patients (see above). Furthermore, the possibility that crowded thoughts could be overrepresented in BP-II should be taken more systematically into account in the diagnostic criteria. It is therefore crucial to better search for and assess thought disturbances in depression in order to guide (or adjust) the most appropriate treatment as early as possible.

\section{Role of funding source}

No funding was provided for this study.

\section{Conflict of interest}

The authors declare that they have no conflict of interest.

\section{Acknowledgment}

The authors thank Mrs. Sandra Ter Pelle for her help in verifying the bibliography.

\section{References}

Akiskal, H.S., Benazzi, F., 2006. Does the FDA proposed list of possible correlates of suicidality associated with antidepressants apply to an adult private practice population? J. Affect. Disord. 94, 105-110.

Akiskal, H.S., Benazzi, F., Perugi, G., Rihmer, Z., 2005. Agitated "unipolar" depression re-conceptualized as a depressive mixed state: implications for the antidepressant-suicide controversy. J. Affect. Disord. 85, 245-258.

American Psychiatric Association, 1980. DSM-III: diagnostic and statistical manual of mental disorders. American Psychiatric Association, Washington.

American Psychiatric Association, 2000. DSM-IV TR: diagnostic and statistical manual of mental disorders. American Psychiatric Association, Washington, DC.

Andreasen, N.C., 1979. Thought, language, and communication disorders. I. Clinical assessment, definition of terms, and evaluation of their reliability. Arch. Gen. Psychiatry 36, 1315-1321.

Arbeitsgemeinschaft für Methodik und Dokumentation in der Psychiatrie (AMDP), 1995. Das AMDP-System: Manual zur Dokumentation psychiatrischer Befunde Hogrefe, Göttingen [etc.].

Aron, A.R., Watkins, L., Sahakian, B.J., Monsell, S., Barker, R.A., Robbins, T.W., 2003. Task-set switching deficits in early-stage Huntington's disease: implications for basal ganglia function. J. Cogn. Neurosci. 15, 629-642.

Association for Methodology and Documentation in Psychiatry (AMDP), Guy (Ed. and translation), W., Ban (Ed. and translation), T.A., 1982. The AMDPSystem: manual for the assessment and documentation of psychopathology. Springer-Verlag, Berlin [etc.]. 
Association pour la méthodologie et la documentation en psychiatrie (AMDP), Preisig (Ed. and translation), M., Serre (Ed. and translation), C., 2000. Le système AMDP: Manuel de documentation de la psychopathologie. Hogrefe, Göttingen [etc.].

Balazs, J., Benazzi, F., Rihmer, Z., Rihmer, A., Akiskal, K.K., Akiskal, H.S., 2006. The close link between suicide attempts and mixed (bipolar) depression: implications for suicide prevention. J. Affect. Disord. 91, 133-138.

Benazzi, F., 2001. Major depressive episodes with hypomanic symptoms are common among depressed outpatients. Compr. Psychiatry 42, 139-143.

Benazzi, F., 2002. Which could be a clinically useful definition of depressive mixed state? Prog. Neuro-psychopharmacol. Biol. Psychiatry 26, 1105-1111.

Benazzi, F., 2003. Depression with racing thoughts. Psychiatry Res. 120, $273-282$.

Benazzi, F., 2005a. Mixed depression: a clinical marker of bipolar-II disorder. Prog. Neuro-psychopharmacol. Biol. Psychiatry 29, 267-274.

Benazzi, F., 2005b. Suicidal ideation and bipolar-II depression symptoms. Hum. Psychopharmacol. 20, 27-32.

Benazzi, F., 2005c. Suicidal ideation and depressive mixed states. Psychother. Psychosom. 74, 61-62.

Benazzi, F., 2005d. Unipolar depression with racing thoughts: a bipolar spectrum disorder? Psychiatry Clin. Neurosci. 59, 570-575.

Benazzi, F., 2006. Symptoms of depression as possible markers of bipolar II disorder. Prog. Neuro-psychopharmacol. Biol. Psychiatry 30, 471-477.

Benazzi, F., 2007a. Bipolar disorder-focus on bipolar II disorder and mixed depression. Lancet 369, 935-945.

Benazzi, F., 2007b. Delineation of the clinical picture of Dysphoric/Mixed Hypomania. Prog. Neuro-psychopharmacol. Biol. Psychiatry 31, 944-951.

Benazzi, F., 2007c. Mixed depression and the dimensional view of mood disorders. Psychopathology 40, 431-439.

Benazzi, F., 2008. A relationship between bipolar II disorder and borderline personality disorder? Prog. Neuro-psychopharmacol. Biol. Psychiatry 32, 1022-1029.

Benazzi, F., Akiskal, H.S., 2001. Delineating bipolar II mixed states in the Ravenna-San Diego collaborative study: the relative prevalence and diagnostic significance of hypomanic features during major depressive episodes. J. Affect. Disord. 67, 115-122.

Benazzi, F., Akiskal, H.S., 2003. The dual factor structure of self-rated MDQ hypomania: energized-activity versus irritable-thought racing. J. Affect. Disord. 73, 59-64.

Benazzi, F., Akiskal, H.S., 2006. Psychometric delineation of the most discriminant symptoms of depressive mixed states. Psychiatry Res. 141, 81-88.

Bertschy, G., Gervasoni, N., Favre, S., Liberek, C., Ragama-Pardos, E., Aubry, J.M., Gex-Fabry, M., Dayer, A., 2007. Phenomenology of mixed states: a principal component analysis study. Bipolar Disord. 9, 907-912.

Bertschy, G., Gervasoni, N., Favre, S., Liberek, C., Ragama-Pardos, E., Aubry, J.M., Gex-Fabry, M., Dayer, A., 2008. Frequency of dysphoria and mixed states. Psychopathology 41, 187-193.

Braden, W., Ho, C.K., 1981. Racing thoughts in psychiatric inpatients. Arch. Gen. Psychiatry 38, 71-75.

Braden, W., Qualls, C.B., 1979. Racing thoughts in depressed patients. J. Clin. Psychiatry 40, 336-339.

Cassidy, F., Forest, K., Murry, E., Carroll, B.J., 1998. A factor analysis of the signs and symptoms of mania. Arch. Gen. Psychiatry 55, 27-32.

Clark, L., Sahakian, B.J., 2008. Cognitive neuroscience and brain imaging in bipolar disorder. Dialogues Clin. Neurosci. 10, 153-163.

Coid, J.W., 1993. An affective syndrome in psychopaths with borderline personality disorder? Br. J. Psychiatry 162, 641-650.

Conway, M., Csank, P.A.R., Holm, S.L., Blake, C.K., 2000. On individual differences in rumination on sadness. J. Pers. Assess. 75, 404-425.

Dale, R.C., Webster, R., Gill, D., 2007. Contemporary encephalitis lethargica presenting with agitated catatonia, stereotypy, and dystonia-parkinsonism. Mov. Disord. 22, 2281-2284.

Drevets, W.C., Price, J.L., Furey, M.L., 2008. Brain structural and functional abnormalities in mood disorders: implications for neurocircuitry models of depression. Brain Struct. Funct. 213, 93-118.

Fink, M., Taylor, M.A., 2001. The many varieties of catatonia. Eur. Arch. Psychiatry Clin. Neurosci. 251 (Suppl 1), I8-13.

Geller, B., Zimerman, B., Williams, M., Delbello, M.P., Bolhofner, K., Craney, J.L., Frazier, J., Beringer, L., Nickelsburg, M.J., 2002. DSM-IV mania symptoms in a prepubertal and early adolescent bipolar disorder phenotype compared to attention-deficit hyperactive and normal controls. J. Child Adolesc. Psychopharmacol. 12, 11-25.

Goodwin, F.K., Jamison, K.R., 1990. Manic-depressive illness. Oxford University Press, New York.

Goodwin, K.F., Jamison, R.K., 2007. Manic-depressive illness: bipolar disorders and recurrent depression. Oxford University Press, New York.

Gu, B.M., Park, J.Y., Kang, D.H., Lee, S.J., Yoo, S.Y., Jo, H.J., Choi, C.H., Lee, J.M., Kwon, J.S., 2008. Neural correlates of cognitive inflexibility during taskswitching in obsessive-compulsive disorder. Brain 131, 155-164.
Guehl, D., Benazzouz, A., Aouizerate, B., Cuny, E., Rotge, J.Y., Rougier, A., Tignol, J., Bioulac, B., Burbaud, P., 2008. Neuronal correlates of obsessions in the caudate nucleus. Biol. Psychiatry 63, 557-562.

Haber, S., 2008. Parallel and integrative processing through the Basal Ganglia reward circuit: lessons from addiction. Biol. Psychiatry 64, 173-174.

Hantouche, E.G., Akiskal, H.S., Azorin, J.M., Chatenet-Duchene, L., Lancrenon, S., 2006. Clinical and psychometric characterization of depression in mixed mania: a report from the French National Cohort of 1090 manic patients. J. Affect. Disord. 96, 225-232.

Hayes, A.E., Davidson, M.C., Keele, S.W., Rafal, R.D., 1998. Toward a functional analysis of the basal ganglia. J. Cogn. Neurosci. 10, 178-198.

Henry, C., Mitropoulou, V., New, A.S., Koenigsberg, H.W., Silverman, J., Siever, L.J., 2001. Affective instability and impulsivity in borderline personality and bipolar II disorders: similarities and differences. J. Psychiatr. Res. 35, 307-312.

Henry, C., Swendsen, J., Van den Bulke, D., Sorbara, F., Demotes-Mainard, J., Leboyer, M., 2003. Emotional hyper-reactivity as a fundamental mood characteristic of manic and mixed states. Eur. Psychiatry 18, 124-128.

Ianzito, B.M., Cadoret, R.J., Pugh, D.D., 1974. Thought disorder in depression. Am. J. Psychiatry 131, 703-707.

Keller, M.B., Lavori, P.W., Coryell, W., Andreasen, N.C., Endicott, J., Clayton, P.J., Klerman, G.L., Hirschfeld, R.M., 1986. Differential outcome of pure manic, mixed/cycling, and pure depressive episodes in patients with bipolar illness. JAMA 255, 3138-3142.

Kirstein, L., Smith, H., 1980. Racing thoughts in primary depression. J. Clin. Psychiatry 41, 199-201.

Koukopoulos, A., 1999. Agitated depression as a mixed state and the problem of melancholia. Psychiatr. Clin. North Am. 22, 547-564.

Kraepelin, E., 1921. Manic-depressive insanity and paranoia. E\&S Livingstone, Edinburgh.

Lawrence, N.S., Williams, A.M., Surguladze, S., Giampietro, V., Brammer, M.J., Andrew, C., Frangou, S., Ecker, C., Phillips, M.L., 2004. Subcortical and ventral prefrontal cortical neural responses to facial expressions distinguish patients with bipolar disorder and major depression. Biol. Psychiatry 55, 578-587.

Leigh, R.J., Foley, J.M., Remler, B.F., Civil, R.H., 1987. Oculogyric crisis: a syndrome of thought disorder and ocular deviation. Ann. Neurol. 22, 13-17.

Mayberg, H.S., 2003. Modulating dysfunctional limbic-cortical circuits in depression: towards development of brain-based algorithms for diagnosis and optimised treatment. Br. Med. Bull. 65, 193-207.

Mayberg, H.S., Liotti, M., Brannan, S.K., McGinnis, S., Mahurin, R.K., Jerabek, P.A., Silva, J.A., Tekell, J.L., Martin, C.C., Lancaster, J.L., Fox, P.T., 1999. Reciprocal limbic-cortical function and negative mood: converging PET findings in depression and normal sadness. Am. J. Psychiatry 156, 675-682.

Meyer, T.D., Hautzinger, M., 2003. The structure of affective symptoms in a sample of young adults. Compr. Psychiatry 44, 110-116.

Nierenberg, A.A., Miyahara, S., Spencer, T., Wisniewski, S.R., Otto, M.W., Simon, N., Pollack, M.H., Ostacher, M.J., Yan, L., Siegel, R., Sachs, G.S., 2005. Clinical and diagnostic implications of lifetime attention-deficit/hyperactivity disorder comorbidity in adults with bipolar disorder: data from the first 1000 STEP-BD participants. Biol. Psychiatry 57, 1467-1473.

Nolen-Hoeksema, S., 1991. Responses to depression and their effects on the duration of depressive episodes. J. Abnorm. Psychology 100, 569-582.

Papageorgiou, C., 2004. Depressive Rumination: Nature, Theory and Treatment. Wiley Publishers, London.

Sachdev, P., 1993. Tardive and chronically recurrent oculogyric crises. Mov. Disord. 8, 93-97.

Salvatore, P., Baldessarini, R.J., Centorrino, F., Egli, S., Albert, M., Gerhard, A., Maggini, C., 2002. Weygandt's on the mixed states of manic-depressive insanity: a translation and commentary on its significance in the evolution of the concept of bipolar disorder. Harv. Rev. Psychiatr. 10, 255-275.

Sato, T., Bottlender, R., Kleindienst, N., Moller, H.J., 2002a. Syndromes and phenomenological subtypes underlying acute mania: a factor analytic study of 576 manic patients. Am. J. Psychiatry 159, 968-974.

Sato, T., Bottlender, R., Kleindienst, N., Tanabe, A., Moller, H.J., 2002b. The boundary between mixed and manic episodes in the ICD-10 classification. Acta Psychiatr. Scand. 106, 109-116.

Sato, T., Bottlender, R., Schroter, A., Moller, H.J., 2003. Frequency of manic symptoms during a depressive episode and unipolar 'depressive mixed state' as bipolar spectrum. Acta Psychiatr. Scand. 107, 268-274.

Sato, T., Bottlender, R., Kleindienst, N., Moller, H.J., 2005. Irritable psychomotor elation in depressed inpatients: a factor validation of mixed depression. J. Affect. Disord. 84, 187-196.

Saxena, S., Rauch, S.L., 2000. Functional neuroimaging and the neuroanatomy of obsessive-compulsive disorder. Psychiatr. Clin. North Am. 23, 563-586.

Serretti, A., Olgiati, P., 2005. Profiles of "manic" symptoms in bipolar I, bipolar II and major depressive disorders. J. Affect. Disord. 84, 159-166.

Whitmer, A.J., Banich, M.T., 2007. Inhibition versus switching deficits in different forms of rumination. Psychol. Sci. 18, 546-553. 
Wilens, T.E., Biederman, J., Wozniak, J., Gunawardene, S., Wong, J., Monuteaux, M., 2003. Can adults with attention-deficit/hyperactivity disorder be distinguished from those with comorbid bipolar disorder? Findings from a sample of clinically referred adults. Biol. Psychiatry 54, 1-8.

Wingo, A.P., Ghaemi, S.N., 2007. A systematic review of rates and diagnostic validity of comorbid adult attention-deficit/hyperactivity disorder and bipolar disorder. J. Clin. Psychiatry 68, 1776-1784.
World Health Organization, 1994. The ICD-10 classification of mental and behavioral disorders: clinical descriptions and diagnostic guidelines. World Health Organization, Geneva.

Yehene, E., Meiran, N., Soroker, N., 2008. Basal ganglia play a unique role in task switching within the frontal-subcortical circuits: evidence from patients with focal lesions. J. Cogn. Neurosci. 20, 1079-1093. 